\title{
Understanding Automatic Behavior in Narcolepsy: New Insights Using a Phenomenological Approach
}

\author{
Michelle Morandin* and Dorothy Bruck
}

Social Sciences and Psychology, Victoria University, Melbourne, Australia

\begin{abstract}
Automatic behaviors are stereotyped, repetitive behaviors performed without awareness and are a common auxiliary symptom of narcolepsy (a disorder of excessive daytime sleepiness). The goal of the study was to expand our knowledge of this complex but poorly understood phenomenon. Here we present new insights based on an analysis of the experiences of ten individuals with narcolepsy. Procedures included two interviews, a family member interview, a one week journal and a one day journal when participants agreed to take minimal or no stimulant medication. Three different types of automatic behavior were identified; sleepiness with low cognitive load, sleepiness with high cognitive load and high cognitive load without sleepiness. Original contributions include: notion of cognitive load, possible progression of automatic behavior to sleep, a new classification of errors, importance of adequate medication, as well as a theoretical discussion.
\end{abstract}

Keywords: Narcolepsy, Automatic behavior, Sleepiness, Vigilance, Cognitive load, Qualitative.

\section{INTRODUCTION}

Narcolepsy is a neurological disorder of excessive daytime sleepiness that is often characterized by several other symptoms; hypnogogic hallucinations, cataplexy, and sleep paralysis [1]. Automatic behavior is a further, poorly understood, symptom with $80 \%$ of individuals with narcolepsy thought to be affected by it $[1,2]$. In European countries narcolepsy has been found to have a prevalence of $0.047 \%$ [3].

Automatic behavior is not exclusive to narcolepsy. A classic example is driving to a destination with little or no recall of the drive. This has been described as 'highway hypnosis' [4] or 'driving without attention mode' [5]. Routine or repeated actions such as driving can become automatized and attention has been shown to shift from external to internal processes. Kerr [5] noted that to the observer the driver may appear to be monitoring their environment (such as checking the speedometer) but internally this information is not being processed. As a result, there is less focus on external feedback and coupled with low levels of alertness, there is increased propensity for errors, failure to act and limited self monitoring [6].

Comparatively little research has examined this phenomenon in people with or without narcolepsy. Early research in the narcolepsy literature described these episodes as 'amnesic states' [7] or 'fugue - like episodes' [8]. Through qualitative analysis, Guilleminault et al. [9] were able to extend the literature to individuals with narcolepsy. It was identified that automatic behavior could occur on a daily basis with durations between a few seconds to several hours.

\footnotetext{
*Address correspondence to this author at the Clinical Neuropsychologist, Epworth Rehabilitation, 888 Toorak Rd, Camberwell, Victoria, 3124, Australia; Tel: (03) 9805 4129; Fax: (03) 9805 4133;

E-mail: Michelle.Morandin@epworth.org.au
}

Tasks often associated with automatic behavior were identified as being monotonous and unskilled. Limited awareness of the automatic behavior was evident until a point in which the skill level required increased. At this time, errors in performance would occur. Guilleminault et al. [10] reported that episodes of automatic behavior often began with individuals feeling sleepy and fatigued, and attempting to fight off drowsiness. In doing so, individuals would find themselves in a state of being less aware of their actions, with deterioration in performance to follow. It was commonly reported that individuals would have complete amnesia of the event or period of time in which it had occurred.

Further documentation of the automatic behavior syndrome was presented in a qualitative study by Zorick et al. [11] of a 55 year old woman with narcolepsy. The woman's most serious episode involved shoplifting. This included theft of large quantities of items, such as jars of pickles, taking tools from a hardware store and taking other items that she had no use for. The authors concluded that these periods involved lapses in awareness.

Unfortunately, the automatic behavior phenomenon in narcolepsy has not been researched further for a number of decades. More importantly, the literature still does not provide a comprehensive understanding of the phenomena. The aim of the current study was to increase our understanding of automatic behavior in individuals with narcolepsy who report experiencing the phenomena. Qualitative methodology was chosen as it allowed an indepth exploration of the phenomena.

\section{METHOD}

\section{Methodological Strategy}

The key principle of the qualitative approach is the belief that the individual has the greatest perspective on the issue at 
Table 1. Participant Details Including Epworth Sleepiness Scale (ESS) Score and Study Completion Data

\begin{tabular}{|c|c|c|c|c|c|c|c|c|c|c|}
\hline Participant & Age & Sex & $\begin{array}{c}\text { Years Since } \\
\text { Narcolepsy } \\
\text { Diagnosis }\end{array}$ & $\begin{array}{c}\text { Experiences } \\
\text { Cataplexy }\end{array}$ & $\begin{array}{c}\text { Off } \\
\text { Medication } \\
\text { ESS score }\end{array}$ & $\begin{array}{c}\text { On } \\
\text { Medication } \\
\text { ESS score }\end{array}$ & $\begin{array}{c}\text { Initial } \\
\text { Interview }\end{array}$ & $\begin{array}{l}\text { Follow-up } \\
\text { Interview }\end{array}$ & Journal 1 & $\begin{array}{c}\text { Journal } 2 \\
\text { (Off Medication) }\end{array}$ \\
\hline A & 69 & M & 12 & $\mathrm{Y}$ & 17 & 5 & $\mathrm{Y}$ & $\mathrm{Y}$ & $\mathrm{Y}$ & $\mathrm{N}$ \\
\hline B & 55 & $\mathrm{~F}$ & 15 & $\mathrm{Y}$ & 22 & 11 & $\mathrm{Y}$ & $\mathrm{Y}$ & $\mathrm{Y}$ & $\mathrm{Y}$ \\
\hline $\mathrm{C}$ & 63 & $\mathrm{~F}$ & 32 & $\mathrm{Y}$ & 24 & 17 & $\mathrm{Y}$ & $\mathrm{Y}$ & $\mathrm{Y}$ & $\mathrm{Y}$ \\
\hline D & 63 & $\mathrm{~F}$ & 17 & $\mathrm{Y}$ & 23 & 10 & $\mathrm{Y}$ & $\mathrm{Y}$ & $\mathrm{Y}$ & $\mathrm{Y}$ \\
\hline E & 66 & F & 26 & $\mathrm{Y}$ & 20 & 13 & $\mathrm{Y}$ & $\mathrm{Y}$ & $\mathrm{N}^{\mathrm{c}}$ & $\mathrm{N}$ \\
\hline $\mathrm{F}^{\mathrm{a}}$ & 46 & M & 10 & $\mathrm{Y}$ & 23 & 19 & $\mathrm{Y}$ & $\mathrm{Y}$ & $\mathrm{Y}$ & $\mathrm{N}$ \\
\hline G & 62 & F & 30 & $\mathrm{Y}$ & 22 & 13 & $\mathrm{Y}$ & $\mathrm{Y}$ & $\mathrm{N}^{\mathrm{c}}$ & $\mathrm{N}$ \\
\hline $\mathrm{H}^{\mathrm{a}}$ & 49 & M & 28 & $\mathrm{~N}^{\mathrm{b}}$ & 22 & 10 & $\mathrm{Y}$ & $\mathrm{Y}$ & $\mathrm{N}^{\mathrm{d}}$ & $\mathrm{N}$ \\
\hline I & 63 & $\mathrm{~F}$ & 40 & $\mathrm{Y}$ & 20 & 9 & $\mathrm{Y}$ & $\mathrm{Y}$ & $\mathrm{N}^{\mathrm{d}}$ & $\mathrm{N}$ \\
\hline $\mathrm{J}$ & 62 & F & 45 & $\mathrm{Y}$ & 22 & 16 & $\mathrm{Y}$ & $\mathrm{Y}$ & $\mathrm{Y}$ & $\mathrm{Y}$ \\
\hline
\end{tabular}

${ }^{\mathrm{a}}$ These participants lived in rural areas. Interviews were conducted over the telephone and the journal sent via post. ${ }^{\mathrm{b}}$ This participant reported not experiencing cataplexy. However they had received a formal diagnosis of narcolepsy. ${ }^{\mathrm{c}}$ These participants were unable to complete the journal as they did not experience automatic behavior during the study. ${ }^{\mathrm{d}}$ These participants did not complete their journals and reported that 'they were unable to find the time to do so'.

hand as they directly deal with the phenomenon, and can therefore provide an account with reference to personal experiences through use of their own words [12]. This approach is of greatest value for research that delves in depth into complex issues with various layers and processes and is rather effective when using small clinical samples, or studying rare phenomenon, in which large scale studies are unable to be conducted [13]. All these points make qualitative research well suited to the subject of the current research.

Phenomenology was selected as the preferred philosophical qualitative approach as it takes the perspectives and interpretations of the individual primarily. Information is gathered through the individual's own descriptions of situations. Information is also taken at different time points and in different situations, to better understand the variables involved [14].

In order to increase the reliability and validity of the study, the principle of triangulation was adopted. Thus multiple methods (i.e. interviews, several types of journals, spouse interviews, ratings) were used to study the same phenomenon.

\section{Participants}

Recruitment focused on the $47 \%$ of people with narcolepsy who reported that they had experienced automatic behaviour (i.e. 22 out of 47) when they completed a questionnaire as part of the research reported in Bruck and Costa [15]. Several other participants were recruited through the support group, the Narcolepsy and Overwhelming Daytime Sleep Society of Australasia Inc. (NODSS) based on their symptom self-report. A short screening questionnaire was used [15] covering the frequency, duration and severity of episodes of automatic behavior and only those reporting current episodes of moderate to severe severity were selected. The final ten participants (three males, seven females) were aged 46-69 years ( $\bar{x}=59.8$, SD $=7.4)$. All met the International Classification of Sleep Disorders (ICSD-2) [16] criteria for narcolepsy with cataplexy $(n=9)$ or narcolepsy without cataplexy $(n=1)$. All had a minimum 10 year history of narcolepsy $(\bar{x}=22.7$ years, $\mathrm{SD}=9.8$ ). Table 1 presents participant details.

\section{Procedures}

Ethics approval for the study was obtained from the Victoria University Psychology Human Research Ethics Committee. Participants indicated their willingness to participate in the study with written consent. The following data collection methods were undertaken and all were completed for each participant within 4 weeks. The sequence of completion was as listed below.

\subsection{Interview One}

Interviews (both one and two) were conducted in the individual's home $(\mathrm{n}=8)$, or, for those living regionally, over the phone $(\mathrm{n}=2)$. The interview had a semi-structured format. Participants were given the freedom to bring up other issues or features of automatic behavior. All interviews were tape recorded with the verbal consent of the participant and transcribed within one week. Below is a list of questions that were asked of participants during the interview:

1. Can you please describe a typical episode of automatic behavior $(\mathrm{AB})$ ?

2. Can you tell me what usually occurs before, during and after an episode of AB?

3. Are there any triggers or signs that an episode of $\mathrm{AB}$ will occur?

4. What usually 'snaps' you out of an episode of AB?

5. Can you control episodes of AB? If so, what strategies do you use to prevent it?

6. Do you find that episodes of $\mathrm{AB}$ are particularly prominent at particular times of the day?

7. What effect does your medication have on AB?

8. Are there any situations that improve or worsen episodes of $\mathrm{AB}$ ? 
9. What effect does sleep deprivation have on your AB?

10. What was your first episode of AB like? Please describe it if you can remember. Did you realize what had just happened? How did you react? What were the consequences of this?

11. Has your AB changed over the years? If so, how?

12. What is the relationship between $\mathrm{AB}$ and the other symptoms of narcolepsy? That is, have you noticed a pattern in the development of your symptoms and how does $A B$ fit in with this?

13. Have episodes of $\mathrm{AB}$ ever stopped for a period of time and then returned? If so, what do you attribute this to?

14. Does $\mathrm{AB}$ interfere with everyday functioning? If so, how?

15. Has an episodes of $\mathrm{AB}$ placed you in a dangerous situation?

16. How do episodes of $\mathrm{AB}$ make you feel, emotionally?

17. How do episodes of AB make you feel, physically?

18. What are the social consequences of $\mathrm{AB}$, now and earlier in your life?

19. What are others reactions to your $\mathrm{AB}$, how do they cope with it?

20. In your experience, how do you explain episodes of $\mathrm{AB}$ ?

\subsection{Journal}

The journal consisted of two sections. Part one was a sleep/wake diary, completed daily for at least one week. It included information regarding the previous night's sleep, naps, medication and food/alcohol intake. Part two was for recording instances of automatic behavior and it included two sections. For the first section of the automatic behavior record, once participants experienced an episode of automatic behavior they were to note the day, time of episode, level of sleepiness before and after the episode, time of last medication, time of last nap and food intake in the last three hours. In order to record their level of sleepiness (both before and after each episode) participants used the Stanford Sleepiness Scale (SSS) [17]. The second section of the automatic behavior record requested a description of what occurred before, during and after the episode, estimated duration, any signs or knowledge that they were having an episode and any comments.

\subsection{Interview Two}

At this meeting/phone call the transcript of the first interview was reviewed to add or clarify responses. A second interview was then conducted to gain an increased depth of information, as individuals may have become more aware of automatic behavior after completing the journal.

\subsection{Family Member Interview}

A spouse or family member was invited to be interviewed and five such interviews were obtained. Interviews were conducted without the presence of the participants and were confidential.

\subsection{Minimal medication journal and follow-up}

On a nominated day (as was convenient) participants took the lowest dosage of stimulant medication that they felt able to take, or no stimulants, and were given a journal (as above) to complete on a day they did not need to leave the house. Only four participants felt able to complete the journal. This was followed up with a brief interview regarding their experiences while keeping the journal. ${ }^{1}$

\subsection{Epworth Sleepiness Scale}

Participants completed the Epworth Sleepiness Scale (ESS) [19]. All participants were currently taking stimulant medication and were asked to complete the 'on medication' ESS about their previous two weeks when on medication. For the ESS 'off medication' they were asked to reflect back on the most recent period when they were off medication and rate their sleepiness in different situations with this in mind. Both types of ratings were completed at the time of the second interview.

\section{Data Analysis}

Step 1. The researcher gained familiarity with the data by conducting numerous readings of the transcripts and journals. Step 2. The process of data reduction (selecting, focusing and simplifying raw data) was undertaken and codes were assigned to the raw data [20]. Step 3. Data was then divided up into groups according to the code and then again coded into subcategories that related to the themes that emerged from statements. Step 4: Data interpretation was then conducted, linking themes and applying meaning to the data. An understanding of a phenomenon was gained by grouping, then conceptualizing statements and regularities, patterns, anomalies and explanations in the coded data were noted [20]. Conceptualizations developed through analyzes of one type of data (e.g. journals) were then checked for congruence with other types of data collected (e.g. interviews). Such multiple cross-examinations of the different materials, and the triangulation of different methods of data collection, increased both the richness of the data and the validity of the findings. The primary researcher was the data coder but themes and conceptualizations were discussed and checked with another researcher, with ambiguities and discrepancies being resolved by consensus.

\section{RESULTS}

The results are presented in terms of three categories that were identified and the key multiple themes that emerged within each. For reasons of space, quotes are only selectively included and more comprehensive documentation of quotes can be found in Morandin [18]. Data from the different types of data collection methods have been integrated in presenting the results. For example, information from the family member interviews may have informed and/or reinforced information about a person's awareness or non-awareness of having experienced an episode or contributed to descriptions

\footnotetext{
${ }^{1}$ The details of the journals and the questions at the second interview and family member interview can be found in Morandin [18].
} 
of different types of errors. [Letters following each quote indicate the participant label].

\section{Perceptions and Behaviors Associated with Automatic Behavior}

\subsection{How Automatic Behavior Events are Perceived}

"For me it's when I'm still going about doing what I'm doing, but my mind is not there" $[\mathrm{H}]$.

All participants consistently reported that all types of automatic behavior were typically associated with a loss of conscious awareness for their actions and subsequently experiencing a 'blank' for that period of time. Sometimes participants described partial awareness of their automatic behavior or regaining full alertness while in the middle of the automatic behavior act.

"I knew I was in automatic behavior because I gradually became aware that I was repeating/going back and forth" [F].

In other cases participants knew they had been in automatic mode because they noticed outcomes, such as missing objects. Further, some participants reported that they would never be aware of some episodes of automatic behavior if they did not have family members or friends point them out.

\subsection{Errors Associated with Automatic Behavior Events}

In many cases, automatic behavior was described as resulting in an unsuccessful activity. Individuals reported that they could often carry on doing a task successfully, and it was not until they were interrupted or an unexpected event arose that the errors in their behavior occurred. This involved errors in sequencing, item/environment intrusions, perseverations leading to nonsense and behaviors that were successful but context inappropriate.

Sequencing errors typically involved omission of a step in a sequence. This included not only missing a step in a behavior, but also mixing up the order of the act.

"I boiled the water up to make spaghetti... I wanted to stir the spaghetti in the water and I actually put my hand in it!" [B].

Item/environment intrusion was associated with individuals wanting to place an item somewhere and continuing with the act of 'putting something away', but mistaking environmental stimuli.

"I could perhaps find a saucepan in the freezer ... your brain carries on doing something, but it can't get it quite right." [D].

There were, however, isolated examples of when items were put in places that were not associated with the desired environmental stimuli and in one case items were consistently placed inappropriately.

"The family's gotten in the habit of asking 'have you looked in your ugg boots ${ }^{2}, \ldots$ on more than one occasion I have lost something and later found it in my ugg boots." [C].

Perseverative actions leading to nonsense involved participants continuing on with the actions of a task in a very

\footnotetext{
${ }^{2}$ An Australian term for a sheep-skinned boot
}

specific manner, such as typing the same letter on a computer over and over again. At times it appeared to others that the individual was functioning appropriately, however once the product of the behavior was examined it became clear that the individual had been in automatic mode. Participants noted that this most frequently involved perseveration on letters, words and topics of conversation.

Context inappropriate behaviors most often occurred when writing or in conversation with others and in some cases were reminiscent of dreaming.

"I mean the whole letter made sense but it just didn't happen..... and I was so embarrassed and I had to pretend that I was writing to someone else. To this day I don't know who it was I was talking about!' [C].

Somewhat similarly, another participant noted that automatic behavior and hallucinations occurred together. Other participants did not report this.

"I was at a restaurant with my husband and I poured lemonade into my plate, instead of pouring it into a glass. But I was hallucinating. And I remember distinctly thinking that that was the glass... so I think I hallucinated at the same time or nearly the same time as the automatic behavior." [J]

Unrecalled actions were those that were successful but appeared to be undertaken while in automatic mode, and it was only by retrospectively looking back and noting that they could not recall a period of time that they knew they had been in automatic mode. This type of automatic behavior was often reported when driving.

\section{Internal States During Automatic Behavior}

Participants described three internal conditions in which automatic behavior would be most likely to occur. All participants described the first two types of automatic behavior which was experienced with sleepiness, while only a subset of three participants described the automatic behavior without sleepiness.

\subsection{Sleepiness with Low Cognitive Load}

This type of automatic behavior was associated with a feeling of sleepiness.

"I do know with me that it happens when I'm fairly tired." [D].

This general feeling of sleepiness was often associated with low stress levels or when completing routine tasks, which often required low cognitive demand/stimulation. It was also often associated with falling asleep. That is, if the individual were placed in a position where sleep would be possible (such as a couch), then the automatic behavior and feeling of sleepiness would progress to complete sleep (this was also indicated by SSS ratings in participant's journals). In these instances individuals described sleep as an interruption to the automatic behavior and that following this they were able to continue on as if they had not just slept.

"Sometimes, I used to eat automatically and then go to sleep while I was eating and then continue on without even being aware that I'd been asleep in the middle of it." [E].

\subsection{Sleepiness with High Cognitive Load}


Similarly, all participants described experiencing automatic behavior with feelings of sleepiness which occurred when completing tasks with high cognitive load. Thus the difference between this and the previous type of automatic behavior was that here participants described feeling pressured to get many tasks completed at such times.

"Stress to get things done tends to have a lot of bearing on the amount of episodes of automatic behavior that I have". [F].

Individuals also described feeling sleepy and being aware of their sleepiness, but pushing on to get the task done. Participants reported that this was sometimes a conscious effort and a desire to complete a task. Alternatively the demands of work and family prevented them from taking the break they needed, and therefore automatic behavior would occur. Participants reported that they believed that if they stopped and had a nap, the automatic behavior would not occur.

The sleepiness was reported by all participants to be largely determined by their stimulant medication intake.

"My episodes of automatic behavior are definitely when my medication time is wearing off." [D].

As a consequence many believed that an effective way to control automatic behavior was through appropriate management of medication. Participants noted that if they did not take their medication appropriately this had an impact on their automatic behavior for that day, and in the days following (a rebound effect). This was also reported to occur when individuals did not take time to rest and sleep when they needed.

"I think it's worse on days following when I've taken insufficient medication." [H].

\subsection{High Cognitive Load Without Sleepiness}

The third instance in which automatic behavior occurred was when participants were pressured to complete a task but felt they were not sleepy or drowsy at the time. This type of automatic behavior was only described by a minority of participants $(\mathrm{n}=3)$.

"I've had automatic behavior when I've been well medicated and thought I was reasonably alert." [C].

It was interesting to note that the participants who experienced this type of automatic behavior had the most severe (highest) ratings on the ESS compared to all other participants in the 'on medication' condition. These participants had 'on-medication' ESS scores at or 16, well within the pathologically sleepy range (see Table 1).

Finally, where automatic behavior involved a high cognitive load, participants believed that it occurred as a type of overload on the system.

"I reckon stress triggers it because it's just like too much for your system to cope with. It just starts to happen whenever you are doing any common thing. After a while it just starts to overload." [B].

\section{Controlling Automatic Behavior}

Participants described attempting many strategies to try and control automatic behavior. These methods are outlined in the following four themes.

\subsection{Controlling the other Symptoms of Narcolepsy}

Three participants reported that by controlling the other symptoms of narcolepsy (particularly daytime sleepiness) the automatic behavior also seemed to be under better control. This was achieved mainly through appropriate medication intake and by following a reasonable sleep pattern.

"If I keep the other symptoms under control um, I don't have as many of the automatic behavior." [F].

\subsection{Stop and Sleep}

Three participants reported that sometimes the only way to prevent an episode of automatic behavior was to stop whatever they were doing and go to sleep. This was applied when individuals were able to recognize that they were very sleepy. However, on many occasions this strategy was not used either because they were unaware of their level of sleepiness or because they did not have the time or opportunity to sleep and needed to get the task done.

\subsection{Develop Habits to Follow}

Two participants reported that rather than develop strategies to prevent episodes, the way they had managed to deal with automatic behavior was by developing habits and relying on routines to minimize the adverse outcomes of the automatic behavior.

"I normally try to develop habits about where I put things, like the car keys. When I come in - they go in a certain place." $[\mathrm{A}]$.

\subsection{Keeping Active}

Three participants reported that remaining active was a way to not only fight sleepiness, but also automatic behavior. Minor or small activities included getting up from a sitting position and carrying out an activity such as getting a drink. Going out into the garden for exercise and deep breathing was also reported as helpful. More dramatic strategies included taking steps to remain in a high state of anxiety and adrenaline. Only one participant reported this. This was achieved by completing tasks at the last minute, running late and changing the order or routine of daily activities.

"I just live on all these funny things like doing things at the last minute... My adrenaline is better than anything, better than Ritalin.'”[B]

\section{DISCUSSION}

The aim of the current study was to develop a further understanding of the automatic behavior phenomenon in individuals with narcolepsy. Several important features of the phenomenon emerged.

All participants consistently reported that automatic behavior was associated with a loss of recall for a period of time as well as reduced awareness of having experienced the episode. These features are not new, with comparative reports also found in the literature $[7,8,10,11]$.

The loss of recall associated with automatic behavior may assist in explaining the subjective memory problems consistently reported with narcolepsy [21], although assessment using objective measures have often been unable to demonstrate a memory deficit $[22,23]$. Objective memory 
tests (involving recall of newly presented materials) have been shown to have limited external validity in relation to everyday memory performance in this group. Based on the current research, it is suggested that automatic behavior may also play a role in this issue.

In cases where automatic behavior resulted in errors, these were reported to occur as a consequence of an increase in the complexity to the task. That is, with the addition of a new step to consider, the individual would either regain full alertness, or continue in automatic mode and make an error in performance. This is consistent with previous research $[10,11]$. Previous research has demonstrated that errors are frequently associated with automatic behavior, however, none have provided a classification of the types of errors that can occur. Rather, a description of the errors they observed was provided, such as that provided by Ganado [8] "they spoke jargon and wrote illegible scribble" (p. 488). The current study was able to define and classify unsuccessful and successful actions in automatic behavior; errors in sequencing, item/environment intrusions, perseverations leading to nonsense and behaviors that were successful but context inappropriate.

A new contribution of the current study is the description of episodes of automatic behavior in terms of the conditions or internal states in which automatic behavior was most likely to occur. Participants described these as three types; sleepiness with low cognitive load; sleepiness with high cognitive load; and high cognitive load without sleepiness. The first two differ in the extent to which sleepiness is accompanied by cognitive load. The low versus high cognitive load across these two types may actually represent a continuum of cognitive load, rather than discrete types. Analysis of the patient interviews and journals suggested the three types of automatic behavior and it should be recognized that the coding process itself may have created a bias toward such categorization. The newly identified automatic behavior without sleepiness (and high cognitive load) requires further consideration, in particular the validation of the report of automatic behavior without low levels of vigilance. Further research could also seek to resolve the issue of whether it is microsleeps or reduced vigilance levels that are associated with automatic behavior accompanied by sleepiness [10, 24].

Both types of automatic behavior with sleepiness were described by all participants and were the types of automatic behavior described in the literature [9]. However, the possible progression of automatic behavior to sleep has not been noted in the literature before. Daniels [7] noted that individuals felt well rested after an episode of automatic behavior but this was not found in the current study. It was more the case that once an individual was feeling alert again the automatic behavior became noticeable. Interestingly, three participants reported instances of automatic behavior in which they did not feel sleepy and this lead to the description of a type of automatic behavior without sleepiness. These episodes were associated with reports of feeling quite alert and reasonably well medicated and occurred with demanding tasks. It is possible that individuals who reported such automatic behavior may have a quite rapid transition, with less awareness, into a low vigilance state and thus experience automatic behavior 'unexpectedly'. Alternatively, they may be operating at a high vigilance level, and it is the high cognitive demands experienced by the individuals which produce this inattentive state.

In normal populations, automatic behavior occurs as a result of slowly diminishing arousal associated with task repetitiveness and, it is hypothesized, an under reliance of external error feedback [4]. However, contrary to narcolepsy, in many cases the level of vigilance is high enough to be able to quickly recognize the behavior and take precautionary actions. For example, it is often easy for individuals without excessive sleepiness to recognize that they are reading the lines of a book without actually taking in the information. However, this recognition is something individuals with narcolepsy find more difficult; their feedback is reduced.

The cognitive mechanism of automatic behavior in narcolepsy would appear to have the same two key components as for people without narcolepsy. In most cases the first component is a state of lower alertness. Across the literature it has been demonstrated that individuals with narcolepsy often have more difficulty performing tasks that are not stimulating, are simple, monotonous, repeated and of long duration compared to challenging tasks conducted in a setting which aids high arousal $[22,23]$. Thus tasks that are repetitive and routine accelerate habituation and/or sleepiness and insufficient medication makes individuals with a disorder of excessive daytime sleepiness especially vulnerable. The second component is a reduced internal awareness of their actions involving a lower responsiveness to feedback. With regard to automatic behavior with high cognitive load, feedback may be especially compromised even when one is relatively alert but overloaded with inputs, or there may be a rapid descent into lower vigilance without awareness which is coupled with reduced feedback.

Some methodological issues require consideration when examining the results. It may be argued that using an older sample (46 - 69 years) is disadvantageous. However, as the study used retrospective data it was essential that the participants were able to reflect on past experiences, and an older sample has more life experience. Further, it may be suggested that being a member of a support group may bias responses in some way. While the current study did use a selective sample (based on the presence of automatic behavior), it was a mixed group in terms of their involvement in the self help group and the reported severity of their narcolepsy. The ten participants involved in this study provided a rich account of this rarely documented phenomenon. It is not claimed that the specific findings are generalizable to the population of people with narcolepsy, only that the increased theoretical understanding of the phenomenon is a valuable addition to the field.

The current study was able to add to the accounts of automatic behavior provided by Daniels [7], Ganado [8], Guilleminault et al. [10] and Zorick et al. [11]. The possibility of automatic behavior leading to sleep has not previously been documented, nor has the important role of adequate stimulant medication to reduce the number of episodes of automatic behavior. Types of errors in automatic behavior have now been classified. Importantly, the notion of cognitive load was not recognized in previous work. 
The findings of the current study provided insights that may assist those living with the condition to have better understanding of their own experiences. Many participants described a sense of feeling like they were psychologically unwell before better understanding the automatic behavior phenomenon. Future research may look at development of classification of mild, moderate or severe cases of automatic behavior with a set of coping strategies for each group. Greater understanding of psychological interventions for individuals with narcolepsy can help to normalize people's experiences and develop greater acceptance of their condition.

The study may have implications not only for individuals with narcolepsy, but also in other groups, such as chronically sleep deprived individuals. It is postulated that the automatic behavior in individuals with and without narcolepsy differs mainly in the level of vigilance and internal awareness of their actions (feedback) when in automatic mode. Thus automatic behavior in sleep deprived individuals may mirror that of individuals with narcolepsy. This hypothesis is consistent with research demonstrating that cognitive functioning following sleep deprivation in individuals without narcolepsy parallels that of the narcolepsy literature [22]. Further research into this area is important as automatic behavior is likely to be a common occurrence in shift workers working at their circadian nadir on routine activities (such as nurses, taxi drivers). Certainty that both lower vigilance levels and higher cognitive load may increase the chance of automatic behaviors (and thus more errors) in those without narcolepsy has implications for preventative measures in shift workers.

\section{BRIEF BIOGRAPHY OF THE RESEARCHER}

Dr. Michelle Morandin completed her Doctorate in Clinical Neuropsychology at Victoria University in 2005, supervised by Professor Dorothy Bruck. Since that time she has gained experience in a number of different areas of neuropsychology including, paediatrics, aged psychiatry, adult mental health, drug and alcohol and traumatic brain injury. She has presented papers at the Australian Society for the Study of Brain Impairment, Worldsleep 2011 and the Australian Sleep Association scientific meetings. She currently works as a Clinical Neuropsychologist at Epworth Rehabilitation in Melbourne with a focus on post traumatic brain injury rehabilitation.

\section{CONFLICT OF INTEREST}

The author(s) confirm that this article content has no conflicts of interest.

\section{ACKNOWLEDGEMENT}

Declared none.

\section{REFERENCES}

[1] Mahowald MW. What is causing excessive daytime sleepiness? Evaluation to distinguish sleep deprivation from sleep disorders. J Postgrad Med 2000; 107: 108-23.

[2] Aldrich MS. Narcolepsy. J Neurol 1992; 42: 34-43.

[3] Ohayon MM, Priest RG, Zulley J, Smirne S, Paiva T. Prevalence of narcolepsy symptomatology and diagnosis in the European general population. Neurology 2002; 58: 1826-33.

[4] Wertheim AH. Highway hypnosis: A theoretical analysis. In: Gale AG, Brown ID, Haslegrave CM, Moorhead I, Taylor S, Eds. Vision in Vehicles III. North-Holland: Elsevier Science Publishers 1991.

[5] Kerr JS. Driving without attention mode (DWAM): A formulation of inattentive states in driving. In: Gale AG, Brown ID, Haslegrave CM, Moorhead I, Taylor S, Eds, Vision in Vehicles III. NorthHolland: Elsevier Science Publishers 1991.

[6] Thiffault P. Bergeron J. Monotony of road environment and driver fatigue: A simulator study. Accid Anal Prev 2003; 35: 381-91.

[7] Daniels LE. Narcolepsy. Medicine 1934; 13: 1-122.

[8] Ganado W. The narcolepsy syndrome. J Neurol 1958; 8: 487-96.

[9] Guilleminault C, Billiard M, Montplaisir J, Dement WC. Altered states of consciousness in disorders of daytime sleep. J Neurol Sci 1975; 26: 377-93.

[10] Guilleminault C, Phillips R, Dement WC. A syndrome of hypersomnia with automatic behavior. Electroencephalogr Clin Neurophysiol 1975; 38: 403-13.

[11] Zorick FJ, Salis PJ, RothT, Kramer M. Narcolepsy and automatic behavior: A case report. J Clin Psychiatry 1979; 40: 194-7.

[12] Denzin NK, Lincoln YS. The handbook of qualitative research. California: Sage Publications 2000.

[13] Bryman A. The debate about quantitative and qualitative research: A question of method or epistemology? Br J Sociol 1984; 35: 7592.

[14] Lemon N, Taylor H. Caring in casualty: The phenomenology of nursing care. In: Hayes $\mathrm{N}$, Ed. Doing qualitative analysis in psychology. East Sussex: Psychology Press 1998.

[15] Bruck D, Costa A. The natural history and symptoms in narcolepsy: A 10 year longitudinal study. Austr J Primary Health 2003; 9: 59-67.

[16] American Academy of Sleep Medicine. The international classification of sleep disorders, diagnostic and coding manual $2^{\text {nd }}$ Ed. Westchester: American Academy of Sleep Medicine 2005.

[17] Hoddes E, Zarcone V, Smythe H, Phillips R, Dement WC. Quantification of sleepiness: A new approach. Int J Psychophysiol 1973; 10: 431-6.

[18] Morandin M. Automatic behavior in individuals with narcolepsy: A qualitative approach [unpublished doctoral dissertation on internet]. Melbourne: Victoria University 2005. Available from http://wallaby.vu.edu.au/adt-VVUT/public/adt-

VVUT20060503.153514/index.html [cited 2010 December 2]

[19] Johns MW. A new method for measuring daytime sleepiness: The Epworth Sleepiness Scale. Sleep 1991; 14: 540-5.

[20] Miles MB, Huberman AM. An expanded sourcebook: Qualitative data analysis, $2^{\text {nd }}$ Ed. London: Sage Publications 1994.

[21] Hood B, Bruck D. Metamemory in narcolepsy. J Sleep Res 1997; 6: 205-10.

[22] Hood B, Bruck D. Sleepiness and performance in narcolepsy. J Sleep Res 1996; 5: 128-34.

[23] Rogers AE, Rosenberg RS. Tests of memory in narcoleptics. Sleep 1990; 13: 42-52.

[24] Valley V, Broughton R. Physiological nature of drowsiness and its relation to performance deficits in narcoleptics. Electroencephalogr Clin Neurophysiol 1983; 55: 243-51. 